\title{
Ansiedad ante exámenes y estrategias de aprendizaje en estudiantes de secundaria de Lima Metropolitana
}

\section{Anxiety in exams and learning strategies in secondary students in Lima Metropolitan}

\author{
Alberto A. Alegre ${ }^{1 a}$ \\ ${ }^{1}$ Universidad San Ignacio de Loyola, Lima, Perú
}

${ }^{a}$ Licenciado en Psicología por la Universidad de Lima y Magister en Neurociencias por la Universidad Nacional Mayor de San Marcos. Especialista en Psicometría. Docente del área de investigación de la Carrera de Psicología en la Universidad San Ignacio de Loyola.

Recibido: $16-05-13$

Aprobado: 06-06-13

\section{Correspondencia}

Email: aalegrebravo@gmail.com

\section{Citar Como:}

Alegre, A. (2013). Ansiedad ante exámenes y estrategias de aprendizaje en estudiantes de secundaria de Lima Metropolitana. Propósitos y Representaciones, 1(1), 107-130. doi: http://dx.doi.org/10.20511/pyr2013.v1n1.9 


\section{Resumen}

El estudio buscó determinar la relación entre la ansiedad ante exámenes y las estrategias de aprendizaje en estudiantes de cuarto y quinto grados de educación secundaria de colegios estatales de Lima Metropolitana. La muestra se obtuvo por un procedimiento intencional, evaluándose a 469 estudiantes, siendo el $45.6 \%$ de sexo masculino y el $54.4 \%$ femenino, pertenecientes a cuatro colegios estatales de Lima Metropolitana. Se evaluó a los estudiantes con el Cuestionario de Ansiedad y Rendimiento - CAR y la Escala de Estrategias de Aprendizaje - ACRA. Se encontró que la ansiedad ante exámenes (puntuaciones en sus tres dimensiones: preocupación, emocionalidad y facilitación) y las estrategias de aprendizaje (puntuaciones en sus cuatro dimensiones: adquisición, codificación, recuperación y apoyo al procesamiento de la información) presentaron correlaciones bajas y en algunos casos negativas; siendo todas estas significativas.

Palabras clave: Ansiedad, ansiedad ante exámenes, estrategias de aprendizaje.

\section{Summary}

This study sought to determine the relationship between test anxiety and learning strategies among students of 4th and 5th grade of secondary of public schools in Lima. The sample was obtained by an intentional method, evaluating 469 students, $45.6 \%$ males and 54.4\% females, belonging to four public schools of Lima. It was used the questionnaire of Anxiety and performance - CAR and the Learning Strategies Scale - ACRA. The test anxiety (punctuations in all three dimensions: concern, emotionality and facilitation) and learning strategies (punctuations in four dimensions: acquisition, coding, support and recovery of information processing) had low correlations and in some cases negative, all of which are significant.

Key words: Anxiety, anxiety tests, learning strategies. 
El estudio de las relaciones entre ansiedad y el rendimiento escolar ha ocupado el interés de psicólogos y educadores durante más de 50 años (Hernández, Pozo, \& Polo, 1994). En general los resultados de la mayor parte de los estudios empíricos al respecto indican que este interés está bien justificado.

La ansiedad como factor individual puede favorecer u obstaculizar el rendimiento en el contexto académico, dependiendo del nivel con que se presenta, la naturaleza de la tarea y el aprendizaje previo del individuo (Crozier, 2001). Sobre el pensamiento acerca de las relaciones entre la ansiedad y el rendimiento han influido dos perspectivas. La primera enfatiza la función organizadora de la ansiedad, que moviliza recursos mentales y físicos para centrar la atención en el origen de la amenaza y descubrir una vía de escape. La segunda hace hincapié en la desorganización. Las teorías psicológicas han tratado de compaginar ambas perspectivas, lo que plantea el problema de especificar las condiciones en las que la ansiedad facilita en rendimiento y en las que lo obstaculiza. La ansiedad o alguno de sus componentes muestran consistentemente relaciones negativas con diversas medidas de rendimiento académico (Edelmann, 1992).

Por otro lado, en el proceso de aprendizaje los estudiantes aplican las denominadas estrategias de aprendizaje, que implican una secuencia de actividades, operaciones o planes dirigidos a la consecución de metas académicas. Estas estrategias tienen un carácter consciente e intencional en el que están implicados procesos de toma de decisiones por parte del alumno, ajustados al objetivo o meta que pretende conseguir. Las estrategias de aprendizaje ponen en relieve dos aspectos importantes: en primer lugar, las actividades $u$ operaciones mentales que realiza el estudiante para mejorar el aprendizaje; en segundo lugar, el carácter intencional o propositivo que implica, por tanto, un plan de acción.

Muchos estudiantes presentan altos niveles de ansiedad ante una evaluación académica, mientras que otros presentan este estado pero en una intensidad baja o moderada, facilitando así una organización y un óptimo desempeño. A partir de lo mencionado, se considera relevante conocer la relación entre las variables ansiedad ante exámenes y estrategias de aprendizaje, pues estas últimas serían factores mediadores, intervinientes 
y moderadores ante la experiencia subjetiva de ansiedad en situaciones de evaluación en el ámbito académico.

Tomando como antecedentes los estudios previos en torno a los constructos en mención (Ávila-Toscano, Hoyos, González \& Cabrales, 2011; Furlan, Sánchez \& Heredia, 2009; Rosario, Núñez, González-Pienda \& Valle, 2008), se propone que las estrategias de aprendizaje para el estudio y comprensión de las materias escolares que emplearían los estudiantes serían factores mediadores ante la frecuencia e intensidad de la experiencia de la ansiedad ante los exámenes.

\section{Ansiedad ante exámenes.}

La ansiedad ante los exámenes se refiere a aquella situación estresante que va generando, desde antes de rendir una prueba y durante la misma, una ansiedad elevada; lo que se deriva en una ejecución deficiente, que trae como consecuencia bajas notas en las asignaturas (Hernández, Pozo, \& Polo, 1994)

Navas (1989) afirma que la ansiedad o temor en las situaciones de examen es generalmente experimentada como una falta de habilidad para pensar en forma clara a pesar de que uno se preparó adecuadamente, hecho que es usualmente independiente del que uno evalúe de forma realista la propia capacidad para salir adelante en esa situación. Papalia (1994) considera la ansiedad ante los exámenes como un factor relacionado con el estrés, que está estrechamente vinculado con la personalidad del individuo. Esta ansiedad afecta la motivación y energía del estudiante mientras realiza la prueba y distrae su atención hacia actividades autodestructivas como la preocupación y la autocrítica. Las personas con una ansiedad desbordada tienen problemas de concentración, a menudo no siguen adecuadamente las instrucciones y desperdician o malinterpretan pistas informativas obvias.

Según Spielberger (1980), la ansiedad ante los exámenes debe considerarse como una forma específica de ansiedad. Las personas muy ansiosas son más propensas a ver los exámenes como situaciones peligrosas o amenazantes, y tienden a preocuparse y llenarse de ansiedad ante dichas situaciones.

Estudios empíricos previos sobre este constructo han demostrado ampliamente la influencia de la ansiedad ante los exámenes en el rendimiento 
académico. Los sujetos que experimentan una alta ansiedad al ser evaluados sufren una merma importante en la ejecución de las pruebas, en comparación con aquellos que no la padecen (Sarason \& Sarason, 1996). Ante esto, Mandler y Sarason (1952) establecieron un primer modelo en el que distinguían dos tipos de impulso: el impulso de tarea que evoca respuestas conducentes a la resolución de la tarea y el impulso de ansiedad que evoca dos tipos de respuesta, las no relacionadas con la tarea (que ejercerían una influencia negativa) y las relacionadas con la tarea (que serían positivas para la solución del problema). La idea general es que las respuestas de ansiedad no relacionadas con la tarea molestan y hacen perder la eficacia a las respuestas de tarea y a las respuestas de ansiedad relacionadas con la tarea. Este déficit se explica a nivel atencional, ya que el individuo no presta la suficiente atención en la resolución de la tarea, debido a la acción de estas respuestas de ansiedad y, por ello, su ejecución es considerablemente entorpecida. Esta explicación se conoce bajo el nombre de hipótesis de interferencia, de gran vigencia en los estudios de la ansiedad ante exámenes (Nicholson, 1958). En ese sentido, Wine (1971) ofrece una interpretación explícita basada en la atención, según la cual se atribuye a los sujetos con alta ansiedad a los exámenes un mayor índice de preocupación, siendo ésta última la que interfiere claramente en la tarea.

Comunian (1993), al estudiar la relación entre la interferencia cognitiva estado-rasgo y la ansiedad ante exámenes, y la influencia de los componentes cognitivos de la ansiedad ante exámenes en el rendimiento escolar, encontró que generalmente varios pensamientos intrusivos y de interferencia se manifestaron en los estudiantes con altos niveles de ansiedad y pobre rendimiento en situaciones de prueba. Mischel, Ebbesen y Zeiss (1972) sostienen que la interferencia de los pensamientos de preocupación tiende a ser máxima cuando la tarea por realizar demanda muchas respuestas. Simplemente es imposible estar lleno de pensamientos negativos acerca de uno mismo y al mismo tiempo concentrarse de manera eficaz en una tarea difícil. En esto coincide Navas (1989), cuando afirma que en el núcleo de la ansiedad existe mucha preocupación y que esta preocupación excesiva o pensamiento irracional impide una ejecución apropiada en los exámenes.

Papalia (1994), al revalorizar el rol de la ansiedad, considera que un aumento moderado de la ansiedad ayuda al estudiante a incrementar el esfuerzo y a concentrar su atención en el examen. Un aumento excesivo, en 
cambio, puede causar estrago en la actuación del alumno. Eso es cierto para la mayoría de los estudiantes de capacidad media. Para esta autora la ansiedad excesiva interfiere minando generalmente las energías del estudiante y su atención hacia actividades cognitivas.

Para comprender la naturaleza y dimensionalidad de la ansiedad ante exámenes, Liebert y Morris (1967), citados por Hernández, Pozo \& Polo (1994) identifican dos componentes fundamentales de la ansiedad ante los exámenes. Uno de marcado carácter cognitivo, "preocupación”, y otro que se refiere a los correlatos fisiológicos de la ansiedad, denominado "emocionalidad", que corresponde a las manifestaciones fisiológicas típicas asociadas a la respuesta de activación del sistema nervioso autónomo. Cuando una persona espera un pobre desempeño la preocupación se incrementa. Sin embargo, en situaciones en las que una persona espera éxito, la preocupación tiende a ser minimizada. Los autores (Hernández, Pozo \& Polo, 1994) indican que la emocionalidad se refiere a las respuestas autonómicas de las personas, las cuales son comunes en situaciones estresantes de evaluación. La ansiedad ante un examen se vuelve emocional cuando el evaluado está sintiendo incertidumbre en la situación inmediata de prueba y la emocionalidad se incrementa cuando se siente inseguro sobre su propio desempeño.

Existen numerosas evidencias de la influencia de la ansiedad ante los exámenes en el rendimiento académico (Cassady \& Johnson, 2002), siendo su componente cognitivo (preocupaciones y pensamientos irrelevantes) el que mayor interferencia puede producir sobre las tareas de codificación, organización, almacenamiento y recuperación de la información, que el estudiante debe realizar cuando se está preparando para un examen o está rindiendo uno.

Si bien la mayoría de teóricos e investigadores desarrollan el constructo en cuestión desde una perspectiva negativa para el rendimiento, autores como Gutiérrez y Avero (1997) acentúan el valor adaptativo de la respuesta de ansiedad. Estos investigadores plantean que si la percepción del nivel del desafío supera el potencial actual para su afrontamiento, se activa un estado de preocupación por la anticipación de un posible fracaso y esto puede movilizar estrategias auxiliares para ampliar los recursos de afrontamiento.

Se han elaborado varios modelos teóricos para explicar la relación entre la ansiedad ante los exámenes y la ejecución en los mismos. Pueden 
agruparse en tres grandes perspectivas: los modelos conductuales, cognitivos y del aprendizaje social. Desde el primer modelo, se supone una inhibición de conductas académicas bajo una situación de castigo, con los componentes condicionados o emocionales habituales en una situación de ansiedad. En cambio, bajo el modelo cognitivo, se incluye la existencia de pensamientos negativos, dificultades en la resolución de problemas, déficits atencionales y baja autoestima, como causas de esta problemática (Blankstein, Flett, Boase \& Toner, 1990). Desde las propuestas enmarcadas en el modelo de aprendizaje social, se incluyen como factores los déficits en habilidades de estudio. Habitualmente se encuentran correlaciones negativas entre una elevada ansiedad y un pobre resultado en el examen, lo que no supone una relación causal sino que se asumen variables mediadoras en las que resultan fundamentales las habilidades de estudio del alumno (Seipp, 1991).

Se han encontrado diferencias entre sexos. Por ejemplo, en los varones un mayor grado de ansiedad está relacionada con una imagen negativa de sí mismo y con unos bajos resultados académicos, mientras que en las mujeres la ansiedad ante los exámenes parece mejorar los resultados en sus notas (Kleijn, Phoeg \& Topmman, 1994). En otros casos, las mujeres informan de una mayor severidad de las respuestas de ansiedad que los hombres, pero esa ansiedad no tiene efecto sobre los resultados de los exámenes (Sud \& Sharma, 1989; Williams, 1994).

\section{Estrategias de aprendizaje.}

En términos conceptuales, las estrategias de aprendizaje pueden definirse, siguiendo a Klauer (1988), como secuencias de acciones dirigidas a la obtención de metas de aprendizaje, por lo que representan complejas operaciones cognitivas que son antepuestas a los procedimientos específicos de la tarea.

Para Weinstein y Mayer (1986), las estrategias de aprendizaje pueden ser definidas como conductas y pensamientos que un aprendiz utiliza durante el aprendizaje con la intención de influir en su proceso de codificación. Para Monereo (2000), son procesos de toma de decisiones (conscientes e intencionales) en los cuales el alumno elige y recupera, de manera coordinada, los conocimientos que necesita para completar una determinada demanda $u$ objetivo, dependiendo de las características de la situación educativa en que 
se produce la acción. Según Genovard y Gotzens (1996), pueden definirse como comportamientos que el estudiante despliega durante su proceso de aprendizaje y que, supuestamente, influyen en los procesos de codificación de la información que se debe aprender.

A partir de estas definiciones, se puede afirmar que existen coincidencias, entre los autores más representativos en este campo, en resaltar algunos elementos importantes del concepto de estrategias de aprendizaje. Por una parte, las estrategias implican una secuencia de actividades, operaciones o planes dirigidos a la consecución de metas de aprendizaje; y por otro, tienen un carácter consciente e intencional en el que están implicados procesos de toma de decisiones por parte del alumno ajustados al objetivo o meta que pretende conseguir. De acuerdo con Beltrán (1996), estas definiciones ponen en relieve dos aspectos importantes. En primer lugar, se trata de actividades $\mathrm{u}$ operaciones mentales que realiza el estudiante para mejorar el aprendizaje. En segundo lugar, las estrategias tienen un carácter intencional o propositivo e implican, por tanto, un plan de acción.

Las estrategias de aprendizaje constituyen actividades conscientes e intencionales que guían las acciones a seguir para alcanzar determinadas metas de aprendizaje. Son actividades potencialmente conscientes y controlables, que están bajo control del estudiante. Es decir, a pesar de que ciertas rutinas pueden ser aprendidas hasta el punto de automatizarse, las estrategias son generalmente deliberadas, planificadas y conscientemente comprometidas en actividades. Dicho en otros términos, las estrategias de aprendizaje son procedimientos que se aplican de un modo intencional y deliberado a una tarea y que no pueden reducirse a rutinas automatizadas. Son más que simples secuencias o aglomeraciones de habilidades. Como afirma Beltrán (1996) las estrategias tienen un carácter intencional, implican por tanto un plan de acción frente a la técnica, que es marcadamente mecánica y rutinaria.

En términos generales, las estrategias de aprendizaje son representadas mentalmente como planes de acción. Borkowsky, Carr y Presssley (1987) han demostrado que la aparente espontaneidad en el uso que una persona hace de las estrategias de aprendizaje es, en realidad, el resultado de una interacción compleja que incluye el conocimiento que tiene de las estrategias de alto nivel 
y sus creencias motivacionales. Desde un punto de vista evolutivo, con la adquisición de estrategias de aprendizaje y de pensamiento específicas para un contexto en diferentes campos de conocimiento, se edifican al mismo tiempo potenciales estrategias más generales. El conocimiento acerca de la estrategia es, al inicio, poco estructurado internamente. En el transcurso del ensanchamiento de la base de conocimiento disponible se debe diferenciar el repertorio de estrategias, tanto que la implementación de estrategias de aprendizaje puede llegar a ser un proceso de adaptación crecientemente flexible.

Los rasgos característicos de las estrategias de aprendizaje son los siguientes: (i) su aplicación no es automática, sino controlada; (ii) implican un uso selectivo de los propios recursos y capacidades disponibles; y, (iii) las estrategias están constituidas de otros elementos más simples, que son técnicas o tácticas de aprendizaje y las destrezas o habilidades.

Si se admite la hipótesis de que los principales aspectos cognitivos del procesamiento de la información son los de adquisición, codificación o almacenamiento y recuperación, las estrategias cognitivas de aprendizaje o estrategias de procesamiento pueden ser definidas como secuencias integradas de procedimientos o actividades mentales que se activan con el propósito de facilitar la adquisición, almacenamiento y/o utilización de la información (Nisbel \& Shucksmith, 1987). Tal hipótesis básica es recogida en el modelo de procesamiento de Atkinson y Shiffrin (1968), en la teoría de los niveles de procesamiento de Craik (1979) y Tulving (1985), también en las teorías acerca de la representación mental del conocimiento en la memoria (Rumelhart \& Ortony, 1977) y en el enfoque "instruccional" (Hernández \& García, 1991; Genovard \& Gotzens, 1996). Estas teorías sostienen que el cerebro funciona como si fuera la condición de tres procesos cognitivos básicos: adquisición, codificación o almacenamiento y recuperación o evocación. Por otra parte, como el pleno rendimiento del sistema cognitivo requiere de la colaboración de otros procesos de naturaleza metacognitiva, oréctica, social, etc., es preciso tener en cuenta aquello que Dansereau (1985) denomina procesos de apoyo.

Por proceso cognitivo entendemos aquella actividad cerebral encargada de transformar, transportar, reducir, coordinar, recuperar o utilizar una "representación mental" del mundo (Neisser, 1976). O parafraseando a 
Sternberg (1982) sería una “operación” realizada sobre una representación interna de objetos o de símbolos, en que los procesos traducen una entrada de información sensorial en una representación conceptual, transforman una representación conceptual en otra, y hasta pueden traducir una representación conceptual en una salida de información motriz.

Aquí interesa señalar que, a partir de los conocimientos disponibles sobre tales procesos cognitivos, se pueden deducir estrategias de procesamiento o estrategias cognitivas para su manipulación (control y dirección). Es decir, procedimientos que permitan optimizar, enseñar, prevenir y/o corregir su adecuado funcionamiento. Estos procedimientos mentales o estrategias de manejo, denominados microestrategias, tácticas de aprendizaje o estrategias de estudio, suelen ser pública o privadamente observables por contraposición a los procesos que son constructos inferidos (Román, 1991).

Para Biggs (1993) el estudio de las estrategias de aprendizaje se sustenta en dos afrontes teóricos: el modelo de procesamiento de la información, que intenta establecer el significado que tienen las estrategias de aprendizaje para el procesamiento de la información; y el modelo orientado al contexto, que se sustenta en el estudio de la fenomenología de la conducta real de estudio y aprendizaje en las instituciones (Yussen, 1985).

Román y Gallego (1994), en base a la hipótesis admitida actualmente sobre los principales procesos cognitivos de procesamiento de la información, plantean que las estrategias cognitivas de aprendizaje o estrategias de procesamiento pueden ser definidas como secuencias integradas de procedimientos o actividades mentales que se activan con el propósito de facilitar la adquisición, almacenamiento y utilización de la información.

Los tipos de estrategias de aprendizaje propuestos por Román y Gallego (1994) son cuatro:

1. Estrategias de adquisición de información. De acuerdo al modelo de Atkinson y Shiffrin (1968), para adquirir información es necesario operar los procesos atencionales, que son los encargados de seleccionar, trasformar y transportar la información desde el medio ambiente al registro sensorial. Luego se ponen en marcha los procesos de repetición, 
encargados de llevar la información (transformarla y transportarla), junto y en interacción con los procesos atencionales, desde el registro sensorial a la memoria a corto plazo.

2. Estrategias de codificación de información. Se refieren al proceso cognitivo encargado de la elaboración y organización de la información, conectándola con conocimientos previos e integrándola en estructuras de significado más amplias que forman la base del conocimiento, es decir, del paso de la memoria de corto plazo a la memoria de largo plazo.

3. Estrategias de recuperación de información. Se refieren a las estrategias que favorecen la búsqueda de información en la memoria y la generación de respuesta.

4. Estrategias de apoyo al procesamiento. Durante el tiempo que dura el procesamiento de información, otros procesos de naturaleza metacognitiva y no-cognitiva, los de apoyo, son neutrales o entorpecen el funcionamiento de las estrategias cognitivas de aprendizaje. Por eso los estudiantes también necesitan estrategias y tácticas que les ayuden a manejar sus estrategias de apoyo. Estas estrategias ayudan y potencian el rendimiento de las estrategias de adquisición, de codificación y de recuperación, incrementando la motivación, la autoestima y la atención. De ahí que para llevar a cabo el procesamiento y recuperación de la información sea imprescindible su identificación y correcto manejo.

\section{Hipótesis.}

Existe relación entre la ansiedad ante exámenes y el uso de las estrategias de aprendizaje en alumnos de cuarto y quinto grado de secundaria pertenecientes a colegios estatales de Lima Metropolitana.

A partir de esta hipótesis teórica se plantearon las siguientes hipótesis específicas:

1. Existe relación entre la ansiedad ante exámenes y el uso de las estrategias de adquisición de la información en alumnos de cuarto y quinto grado de secundaria pertenecientes a colegios estatales de Lima Metropolitana. 
2. Existe relación entre la ansiedad ante exámenes y el uso de las estrategias de codificación de la información en alumnos de cuarto y quinto grado de secundaria pertenecientes a colegios estatales de Lima Metropolitana.

3. Existe relación entre la ansiedad ante exámenes y el uso de las estrategias de recuperación de la información en alumnos de cuarto y quinto grado de secundaria pertenecientes a colegios estatales de Lima Metropolitana.

4. Existe relación entre la ansiedad ante exámenes y el uso de las estrategias de apoyo al procesamiento en alumnos de cuarto y quinto grado de secundaria pertenecientes a colegios estatales de Lima Metropolitana.

\section{Método}

El método utilizado fue el descriptivo correlacional (Hernández, Fernández \& Baptista, 2003), con el propósito de evidenciar y describir las relaciones entre los puntajes de las variables (ansiedad ante exámenes y estrategias de aprendizaje) en los alumnos de cuarto y quinto grado de secundaria pertenecientes a colegios estatales de Lima Metropolitana. El diseño fue de tipo descriptivo correlacional bivariado, buscando determinar las relación entre las variables de estudiadas (Sánchez \& Reyes, 2002).

\section{Participantes.}

La población objetivo estuvo constituida por todos los alumnos de cuarto y quinto grado de secundaria pertenecientes a colegios estatales de Lima Metropolitana. La muestra del estudio se obtuvo por un procedimiento intencional no probabilístico, eligiéndose a estudiantes de cuatro colegios estatales mixtos con nivel secundario que poseían turnos de mañana y tarde.

La muestra total estuvo conformada por 469 alumnos del cuarto y quinto grado de nivel secundaria, $232(49.5 \%)$ pertenecientes al cuarto y $237(50.5 \%)$ al quinto respectivamente. Según la variable sexo, 214 (45.6\%) estudiantes pertenecen al sexo masculino y $255(54.45 \%)$ al femenino (véase tabla 1). 
Tabla 1.

Composición de la muestra

\begin{tabular}{lcc}
\hline & Frecuencia & Porcentaje \\
\hline Sexo & 214 & 45.6 \\
Masculino & 255 & 54.4 \\
Femenino & & \\
Grado & 232 & 49.0 \\
Cuarto & 237 & 50.5 \\
Quinto & & \\
Edad & 1 & 0.2 \\
13 & 52 & 11.1 \\
14 & 156 & 33.3 \\
15 & 173 & 36.9 \\
16 & 72 & 15.4 \\
17 & 13 & 2.8 \\
18 & 2 & 0.4 \\
19 & & \\
\hline
\end{tabular}

$N=469$

\section{Variables de estudio.}

- Ansiedad ante exámenes. Cuantificada a través del Cuestionario Ansiedad y Rendimiento - CAR, que evalúa la ansiedad ante el rendimiento (Ferrando, Varca \& Lorenzo, 1999).

- Estrategias de aprendizaje. Evaluada a través de la Escala de Estrategias de Aprendizaje - ACRA (Román \& Gallego, 1994).

\section{Instrumentos.}

\section{Cuestionario de Ansiedad y Rendimiento - CAR.}

El marco teórico general en el que se desarrolla el CAR es el de la ansiedad de tarea, la ansiedad como facilitadora del rendimiento y la ansiedad como perturbadora del rendimiento. 
De acuerdo con los principios teóricos del papel de la ansiedad como componente implicado en el rendimiento académico y su vasta evidencia empírica, el CAR fue diseñado para tener una estructura factorial tridimensional, es decir, medir los componentes de preocupación, emocionalidad y ansiedad facilitadora, como factores interrelacionados (Ferrando, Varca \& Lorenzo, 1999).

Los ítems del CAR se refieren a situaciones imaginadas de evaluación y pretenden ser un reflejo del pensamiento verbal del sujeto en situación de examen. El CAR contiene en total de 30 ítems dicotómicos, distribuidos de la siguiente forma:

- Preocupación: del ítem 1 al 15.

- Emocionalidad: del ítem 16 al 24.

- Ansiedad Facilitadora: del ítem 25 al 30.

En esencia, el CAR es una prueba específica y situacional, de carácter cognoscitivo y de alcance restringido. Para el estudio se analizaron las propiedades psicométricas de las puntuaciones obtenidas por la muestra en el instrumento. La confiabilidad se estimó mediante el análisis de la consistencia interna, obteniéndose un coeficiente de confiabilidad alfa de Cronbach de .089 para la escala completa; en tanto que se obtuvieron coeficientes de $0.91,0.77$ y 0.83 para las dimensiones de preocupación, emocionalidad y ansiedad facilitadora, respectivamente. Para obtener evidencias de validez se analizó la estructura interna del instrumento a partir de las puntuaciones de los evaluados mediante el análisis factorial exploratorio, empleando el método de componentes principales con rotación varimax. Se encontraron tres factores con autovalores mayores a uno (criterio de Kaiser) y que en su conjunto explican el $70.476 \%$ de la varianza acumulada: Por otro lado, la matriz factorial rotada evidenció la estructura de tres factores con cargas factoriales mayores a 0.40 .

\section{Escala de Estrategias de aprendizaje-ACRA.}

El uso de las estrategias de aprendizaje se evaluó a través de las ACRA (Román \& Gallego, 1994). Se trata de cuatro escalas independientes que evalúan el uso que habitualmente hacen los estudiantes de las estrategias de 
aprendizaje. La escala I evalúa las estrategias de adquisición de información: atencionales y de repetición. La escala II evalúa las estrategias de codificación o almacenamiento de información: mnemóticas, de elaboración y de organización. La escala III evalúa las estrategias de recuperación de información: búsqueda y generación de respuesta. La escala IV evalúa las estrategias de apoyo al procesamiento: autoconocimiento, automanejo, autoinstrucciones, interacciones sociales, motivación intrínseca, extrínseca y de escape.

Los enunciados se valoran en un sistema Likert, con cinco puntos de calificación, que van desde nunca $(\mathrm{O})$ hasta siempre (5), donde los puntajes por escala se obtienen sumando los ítems respectivos. Las ACRA están diseñadas para ser administradas en 50 minutos, a grupos de adolescentes o adultos.

Se revisaron las propiedades psicométricas de las cuatro escalas, a partir de las puntuaciones obtenidas por la muestra en cada una de ellas. La confiabilidad se calculó analizando la consistencia interna de las puntuaciones, obteniéndose como coeficientes de confiabilidad Alfa de Cronbach de 0.85, $0.93,0.78$ y 0.88 , para las escalas I, II, III y IV, respectivamente. La validez de cada escala se estableció a través del análisis factorial exploratorio, por el método de componentes principales con rotación varimax. Se encontró un solo factor en cada escala. Los factores extraídos presentaron autovalores mayores a cuatro y cada escala independientemente explicaba más del $40.869 \%$ de la varianza total, lo que evidencia en cada escala la unidimensionalidad.

\section{Resultados}

\section{Análisis descriptivo.}

Los resultados del análisis de la bondad de ajuste a la curva normal, realizado a través de la prueba Kolgomorov-Smirnov (véase tabla 2), indican que los puntajes obtenidos en las cuatros dimensiones de la ansiedad ante exámenes no tienen una distribución normal, mientras que en las cuatro escalas de estrategias de aprendizaje si se presenta dicha distribución. Es debido a estos resultados que los análisis estadísticos de los datos son del tipo no paramétrico. 
Tabla 2.

Análisis de la bondad de ajuste a la curva normal de Kolgomorov-Smirnov

\begin{tabular}{|c|c|c|c|c|c|}
\hline Variables & Dimensiones & M & D.E. & $\begin{array}{l}Z \text { de Kol- } \\
\text { mogorov- } \\
\text { Smirnov }\end{array}$ & $\begin{array}{c}\mathrm{p} \\
\text { a dos } \\
\text { colas }\end{array}$ \\
\hline Ansiedad ante & Preocupación & 6.70 & 2.95 & 1.78 & .003 \\
\hline \multirow[t]{4}{*}{ Exámenes } & Emocionalidad & 3.81 & 2.09 & 2.35 & .000 \\
\hline & Ansiedad facilitadora & 3.72 & 1.28 & 3.63 & .000 \\
\hline & Adquisición & 46.70 & 8.37 & 1.10 & .175 \\
\hline & Codificación & 109.59 & 20.18 & 0.85 & .460 \\
\hline \multirow[t]{2}{*}{$\begin{array}{c}\text { Estrategias de } \\
\text { aprendizaje }\end{array}$} & Recuperación & 46.93 & 8.91 & 1.09 & .183 \\
\hline & Apoyo al procesamiento & 93.18 & 15.55 & 1.14 & .152 \\
\hline
\end{tabular}

$\mathrm{N}=469$

\section{Contrastación de hipótesis.}

Inicialmente se planteó la hipótesis teórica, que proponía que existe relación entre la ansiedad ante exámenes y las estrategias de aprendizaje en alumnos de cuarto y quinto grado de secundaria pertenecientes a colegios estatales de Lima Metropolitana. Para contrastarla se efectuó el análisis a través del coeficiente de correlación de Spearman (rho).

Al analizar la tabla 3 se puede observar que la ansiedad ante exámenes (puntuaciones en sus tres dimensiones) y las estrategias de adquisición de la información tienen correlaciones bajas y significativas, siendo dos de ellas negativas como son los casos de las correlaciones entre "preocupación" y "emocionalidad" con las "estrategias de adquisición" ( $\mathrm{r}=-.151$ y r $=$ .121). Por tanto, se concluye que la primera hipótesis específica, en la cual se planteó que existe relación entre la ansiedad ante exámenes y el uso de las estrategias de adquisición de la información, se puede aceptar. Sin embargo, es importante resaltar que la asociación entre ambas variables es débil. 
Tabla 3 .

Correlación de Spearman entre la ansiedad ante exámenes y las estrategias de adquisición de la información

\begin{tabular}{lcc}
\hline Correlación & $\mathrm{R}$ & $\mathrm{p}$ \\
\hline Preocupación - Estrategias de adquisición & -.151 & .001 \\
Emocionalidad - Estrategias de adquisición & -.121 & .009 \\
Ansiedad facilitadora - Estrategias de adquisición & .266 & .000 \\
\hline $\mathrm{p}<.05$ & & \\
$\mathrm{~N}=469$ & &
\end{tabular}

En los alumnos de cuarto y quinto grados de secundaria las correlaciones entre las puntuaciones en las dimensiones de la ansiedad ante exámenes (emocionalidad, preocupación y ansiedad facilitadora) y las estrategias de codificación de la información son bajas $(\mathrm{r}=-.310)$, negativas $(\mathrm{r}=-.174$ y $\mathrm{r}=-.158$ ) y significativas (véase tabla 4), lo cual indica que la segunda hipótesis se acepta, pero con una relación mínima entre las variables.

Tabla 4.

Correlación de Spearman entre la ansiedad ante exámenes y las estrategias de codificación de la información

\begin{tabular}{lll}
\hline Correlación & $\mathrm{R}$ & $\mathrm{p}$ \\
\hline Preocupación - Estrategias de codificación & -.174 & .001 \\
Emocionalidad - Estrategias de codificación & -.158 & .001 \\
Ansiedad facilitadora - Estrategias de codificación & .310 & .000 \\
\hline
\end{tabular}

$\mathrm{p}<.05$

$N=469$

Las relaciones entre la ansiedad ante exámenes (puntuaciones en las dimensiones) y las estrategias de recuperación de la información (véase tabla 
5), son significativas y bajas, por lo que la tercera hipótesis es aceptada, aunque la relación entre las variables sea débil.

Tabla 5.

Correlación de Spearman entre la ansiedad ante exámenes y las estrategias de recuperación de la información

\begin{tabular}{lcc}
\hline Correlación & $\mathrm{R}$ & $\mathrm{p}$ \\
\hline Preocupación - Estrategias de recuperación & -.210 & .000 \\
Emocionalidad - Estrategias de recuperación & -.156 & .001 \\
Ansiedad facilitadora - Estrategias de recuperación & .211 & .000 \\
\hline
\end{tabular}

$\mathrm{p}<.05$

$\mathrm{N}=469$

La hipótesis en la que se establece relación entre la ansiedad ante exámenes y las estrategias de apoyo al procesamiento de la información es aceptada. Así, en el análisis de las puntuaciones obtenidas por la muestra se observa que las asociaciones entre ambas variables son bajas y significativas (véase tabla 6).

Tabla 6.

Correlación de Spearman entre la ansiedad ante exámenes y las estrategias de apoyo al procesamiento de la información

\begin{tabular}{lcc}
\hline Correlación & $\mathrm{R}$ & $\mathrm{p}$ \\
\hline $\begin{array}{l}\text { Preocupación - Estrategias de apoyo al procesamiento de la } \\
\text { información }\end{array}$ & -.180 & .000 \\
$\begin{array}{l}\text { Emocionalidad - Estrategias de apoyo al procesamiento de la } \\
\text { información. }\end{array}$ & -.152 & .001 \\
$\begin{array}{l}\text { Ansiedad facilitadora - Estrategias de apoyo al procesamiento de } \\
\text { la información }\end{array}$ & .260 & .000 \\
\hline
\end{tabular}

$p<.05$

$\mathrm{N}=469$ 


\section{Discusión}

Los resultados obtenidos en el presente estudio permiten reafirmar el principio que para comprender la elevada ansiedad de los estudiantes durante los exámenes, es preciso analizar las estrategias de aprendizaje que los estudiantes utilizan durante la fase de preparación (Cassady \& Johnson, 2002). A pesar de que las correlaciones son débiles, se ha corroborado que a mayor puntuación en los componentes de la ansiedad, preocupación y emocionalidad, menor eficacia en el uso de estrategias de aprendizaje. Mientras que la ansiedad facilitadora se relaciona de forma positiva con el uso de dichas estrategias. Los modos de apropiación del conocimiento en los estudiantes evaluados se asocian al uso preferencial de unas u otras estrategias para adquirir, codificar, recuperar y procesar metacognitivamente la información en la fase previa y durante las situaciones de evaluación académica (Hernández, Pozo \& Polo, 1994). Estos resultados coinciden con lo reportado por Escurra (2004) y Román (2004), de manera que es factible afirmar que los escolares estudiados que conocen y hacen uso de tácticas para aprender, emplean también de forma selectiva las estrategias para modular los estados afectivos que limitan su desempeño en situaciones de evaluación.

Estos resultados deberían ser considerados en los planes de estudio de educación secundaria, para que a través de intervenciones pedagógicas se desarrollen en los estudiantes las estrategias de aprendizaje y de preparación frente a exámenes, con el propósito de que los alumnos ante la experiencia evaluativa puedan articular y emplear estratégicamente técnicas para adquirir, almacenar y recuperar información académica, y así mejorar su rendimiento académico (Ávila-Toscano, Hoyos, González \& Cabrales, 2011). Por lo tanto, las instituciones educativas deberían diseñar programas para desarrollar estrategias de aprendizaje de preparación ante los exámenes, en especial en los primeros grados del nivel secundario, e implementar programas de control y modulación de la ansiedad frente a la evaluación. Para lograr esto se requeriría de la competencia didáctica del docente, de un currículo adecuado y el empleo de las nuevas tecnologías de la información.

Además, sería necesario discutir sobre las características de los procedimientos evaluativos que se emplean en los diferentes contextos institucionales de los que se extrajo la muestra de estudiantes. Esto 
con la finalidad de establecer el énfasis en la adquisición estratégica de conocimientos y no sólo en la mera reproducción. Este énfasis se asocia a la elección de estrategias que el alumno tenderá a percibir como más adecuadas para el tipo de consignas a las que deba responder en la evaluación.

Los programas escolares debieran contemplar, en principio, una instancia de revisión de estrategias de aprendizaje que los alumnos utilizan habitualmente, a fin de estimar el tipo de apropiación de conocimientos que ellos logran. Además, debieran promover el uso de estrategias de apoyo al procesamiento de la información (estrategias metacognitivas y socio-afectivas), para así desarrollar tácticas que les ayuden a potenciar el rendimiento de las estrategias de adquisición, de codificación y de recuperación, contribuyendo así a la disminución de la ansiedad ante exámenes. Finalmente, toda intervención pedagógica debe generar el clima propicio y adecuado para un funcionamiento integrado del sistema cognitivo del estudiante, posibilitándole controlar, canalizar o reducir la ansiedad, los sentimientos de incompetencia, las expectativas de fracaso, etc., como variables relacionadas e implicadas en la ansiedad ante los exámenes y por tanto en el rendimiento académico (Quintana, 1998).

\section{Conclusiones}

Los resultados de la investigación permiten llegar a las siguientes conclusiones:

- Existe relación entre la ansiedad ante exámenes y el uso de las estrategias de adquisición de la información en los alumnos de cuarto y quintos grado de secundaria pertenecientes a colegios estatales de Lima Metropolitana.

- Existe relación entre la ansiedad ante exámenes y el uso de las estrategias de codificación de la información en dichos alumnos.

- Existe relación entre las variables ansiedad ante exámenes y empleo de estrategias de recuperación de la información en dichos alumnos, debido a que el coeficiente de correlación alcanzado es significativo.

- Existe relación entre la ansiedad ante exámenes y el uso de estrategias de apoyo al procesamiento de la información en dichos alumnos. 


\section{Referencias}

Atkinson, R. \& Shiffrin, R. (1968). Human memory: a proposed system and its control processes. The psychology of learning and motivation: Advances in research and theory, 2, 89-195.

Ávila-Toscano, J., Hoyos, S., González, D., \& Cabrales, A. (2011). Relación entre ansiedad ante los exámenes, tipo de pruebas y rendmiento académico en estudiantes universitarios. Psicogente , 14(26), 255-268.

Beltrán, J. (1996). Estrategias de aprendizaje. En Beltrán, J. \& Genovard, C. (Eds.). Psicología de la instrucción. Variables y procesos básicos (pp. 63-82). Madrid: Síntesis.

Biggs, J. (1993). What do inventories of student's learning processes really measure? A theoretical review and clarification. British Journal of Educational Psychology, 63, 3-19.

Blankstein, K., Flett, G., Boase, P., \& Toner, B. (1990). Thought listing and endorsement measures of self-referential thinking in test anxiety. Anxiety Research, 2, 103-111.

Borkowsky, J., Carr, M. \& Pressley, M. (1987). Spontaneous Strategy Use: Perspectives from Metacognitive Theory. Intelligence, 11, 61-75.

Cassady, J. \& Johnson, R. (2002). Cognitive test anxiety and academic performance. Contemporary Educational Psychology, 27, 270-295.

Comunian, J. (1993). Anxiety, cognitive interference and school performance of Italian children. Psychological Reports, 73, 747-754.

Craik, F. (1979). Elaboration and distinctiveness in episodic memory. Journal of verbal Learning and Verbal Behavior. 11, 671-684.

Crozier, R. (2001). Diferencias individuales en el aprendizaje. Narcea: Madrid.

Dansereau, D. (1985). Learning strategies research. En Segal, J. (Ed.), Thinking and learning skills (pp. 48-66). Hillsdale: Erlbaum.

Edelmann, R. (1992). Anxiety: Theory, Research and Intervention in Clinical and Health Psychology. Inglaterra: Wiley.

Escurra, M. (2004). Influencia de las estrategias de aprendizaje y la reflexión activa sobre el rendimiento escolar en los alumnos del quinto año de 
secundaria de la ciudad de Lima. Revista de Investigación PsicológicaUniversidad Nacional Mayor de San Marcos, 7, 51-80.

Ferrando, P., Varca, D. \& Lorenzo, U. (1999). Evaluación Psicométrica del Cuestionario de Ansiedad y Rendimiento en una muestra de escolares. Psicothema, 11, 225-236.

Furlan, L., Sánchez, J., \& Heredia, D. (2009). Estrategias de aprendizaje y ansiedad ante los exámenes en estudiantes universitarios. Pensamiento psicológico, 4, 117-124.

Genovard, C. \& Gotzens, C. (1996). Psicología de la instrucción. Variables y procesos básicos. Madrid: Síntesis.

Gutiérrez, M. \& Avero, P. (1997). Ansiedad, estrategias auxiliares y comprensión lectora: Déficit de procesamiento versus falta de confianza. Psicothema, 7, 569-578.

Hernández, P. \& García, L. (1991). Psicología y enseñanza del estudio: teorías y técnicas para potenciar las habilidades intelectuales. Madrid: Pirámide.

Hernández, R., Fernández, C. \& Baptista, P. (2003). Metodología de la Investigación ( $5^{\mathrm{a}}$ ed.). México: McGraw Hill.

Hernández, J., Pozo, C. \& Polo, C. (1994). Ansiedad ante los exámenes: Un programa para su afrontamiento eficaz. Valencia: Promolibro Valencia.

Klauer, K. (1988). Teaching for learning to learn. A critical appraisal with some proposal. Instructional Science, 17, 351-367.

Kleijn, W., Ploeg, H. \& Topman, R. (1994). Cognition, study habits, test anxiety, and academic performance. Psychological Reports, 75, 1219-1226.

Mandler, G. \& Sarason, S. (1952). A study of anxiety and learning. Journal of abnormal and social psychology, 47, 166-173.

Mischel, W., Ebbesen, E. \& Zeiss, A. (1972). Cognitive and attentional mechanisms in delay of gratification. Journal of Personality and Social Psychology, 21, 204-696.

Monereo, C. (2000). Estrategias de Aprendizaje. Madrid: Visor Dis. S.A.

Navas, J. (1989). Ansiedad en la toma de exámenes: Algunas explicaciones cognoscitivas y comportamiento. Revista de aprendizaje $y$ comportamiento, $7,21-41$. 
Neisser, U. (1976). Psicología Cognoscitiva. México: Trillas.

Nicholson, W. (1958). The influence of anxiety upon learning. Journal of Personality, 26, 303-319.

Nisbel, J. \& Schucksmith, J. (1987). Estrategias de Aprendizaje. Madrid: Santillana.

Papalia, D. (1994). Psicología. Madrid: McGraw-Hill.

Quintana, A. (1998). Afrontamiento del estrés frente al examen en educación superior. (Tesis de maestría inédita). Universidad Nacional Mayor de San Marcos.

Román, S. \& Gallegos, R. (1994). ACRA - Escala de Estrategias de Aprendizaje. ( $2^{\mathrm{a}}$ ed.). Madrid: TEA Ediciones S.A.

Román, S. (2004). Procedimiento de aprendizaje autorregulado para universitarios: La estrategia de lectura significativa de textos. Revista electrónica de investigación psicoeducativa, 2, 113-132. Recuperado el 5 de agosto de 2010, de http://www.investigacion-psicopedagogica.org/revista

Román, S. (1991, 15 octubre). Análisis diferencial de estrategias de aprendizaje en educación secundaria. Conferencia presentada en el Congreso Internacional sobre Intervención Psicoeducativa. Madrid: Universidad Complutense de Madrid.

Rosario, P., Núñez, J. S., González-Pienda, J., \& Valle, A. (2008). Ansiedad ante los exámenes: relación con variables personales y familiares. Psicothema , 20(4), 563-570.

Rumelhart, D. \& Ortony, A. (1977). The representation of knowledge in memory. New Jersey: Hillsdale.

Sánchez, H. \& Reyes, C. (2002). Metodología y Diseños en la Investigación Cientifica. Lima: Editorial Universitaria.

Sarason, I. \& Sarason B. (1996). Psicología anormal. El problema de la conducta inadaptada. México: Printice-Hall.

Seipp, B. (1991). Anxiety and academic performance: A meta-analysis of findings. Anxiety Research, 4, 27-41.

Spielberger, Ch. (1980). Tensión y Ansiedad. México: Harper \& Row Latinoamericana. 
Sternberg, R. (1982). Inteligencia Humana: Cognición. Madrid: Paidós.

Sud, A. \& Sharma, H. (1989). Test anxiety, intrusive thoughts and attentional process. Journal of Personality and Clinical Studies, 5, 139-145.

Tulving, E. (1985). How many systems are there?. American Psychologist, 40, 385-398.

Weinstein, C. \& Mayer, R. (1986). The teaching of learning strategies. En: Wittrock M. (Eds). Handbook of research on teaching ( $3^{\mathrm{a}}$ ed.). Nueva York: MacMillan Publishing Company.

Wine, J. (1971). Test anxiety and direction of attention. Psychological Bulletin, 76, 92-104.

Williams, J. (1994). Anxiety measurement: Construct validity and test performance. Measurement and Evaluation in Counseling and Development, 27, 302-307.

Yussen, S. (1985). El rol de la metacognición en las teorías contemporáneas del desarrollo cognitivo. En Forrest-Presley, D., MackKinnon, G. \& Gary Weller, F. (Eds.), Metacognición, cognición y performance humana. Orlando: Academic Press. 